\title{
No-moving-part fluidic terminal units for control of thermal comfort in buildings (a survey)
}

\author{
Václav Tesařl,* \\ ${ }^{I}$ Institute of Thermomechanics v.v.i., Czech Academy of Sciences, Dolejškova 5, 18200 Praha-Kobylisy, Czech Republic
}

\begin{abstract}
This article surveys a so far little known approach to two simultaneously requested but mutually opposed requirements on internal conditions in buildings. It is the growing demand of better internal comfort while the other is decrease of costs and energy consumption. The internal conditions is ensured by terminal units located and adjusted at the points of conditioned air delivery into rooms. Author's terminal units achieve the goal by having no moving parts. Advantages are low cost, robustness and long operating life without maintenance. Capability to switch the air flows at a high frequency without wear opens new ways to control, such as the flow pulse width modulation. The fast switching also makes possible small regenerative heat exchangers, keeping inside the building the heat extracted from the removed contaminated air.
\end{abstract}

\section{Introduction}

Buildings may seem to be not particularly important consumers of energy. In fact maintaining the living conditions consumes as much as $\sim 40 \%$ of total worldwide energy production - and this proportion rapidly grows. The reason is not only the accelerated expansion of new buildings construction but also increasing demands on quality and comfort [13]. The main part of the energy consumed in a building is spent on adjusting temperature - by heating in winter, especially in cold climates, and cooling in summer and hot regions. It was demonstrated already a long time ago that producing the heat (or cold) locally in each room of the building is widely uneconomical. Of course, the heat or cold produced in a central plant requires transporting through a ductwork to meet local needs - and this has also its inherent problems.

What currently adds considerably to the increasing energy consumption in buildings are growing occupants' demand on thermal comfort. Meeting the requirements mainly means applying the temperature control separately in each occupants' locations. This is due to problems with what particular occupant asks for. The requests are influenced by particular individual factors like their instantaneous (and also previous) physical effort, racial background, or even instantaneous mental state. To satisfy the various local needs in rooms with more than one occupant leads to permanent manipulations with local controllers. Also rooms nominally identical have different demands due to variables like positions of partitions, cubicles, cabinets, furniture, and also the incident solar radiation of widows and walls and its variation during the day. This all calls for thermal control applied in the terminal units which deliver to the occupants the warm or cold air — which is, in addition, processed by filtering, humidity removal (or humidity addition, e.g. in frosty winters). Currently rapidly increasing in demand is personalised ventilation. It means delivering fresh air to a terminal unit directly at each occupant's desk, bed, or work place. Standard present-day versions of terminal units are no more satisfactory. They have a high first cost. Because the mechanical moving components with which they operate, usually electric motor of blower drives and moving flow control flaps, cause also quite high operation costs because they need maintenance.

Meeting the mutually opposing requests of comfort and yet low cost and low energy demand are the currently developed new fluidic terminal units. They have no mechanical movable parts, using the principles of pure fluidics. They may be manufactured at a low cost by advanced methods like the 3D printing (with absence of expensive manual operations in production and assembly) and offer long lifetime without maintenance. The idea of fluidics brings also other secondary advantages, such as the reduced number of electric conductors decreasing the danger of fires, etc..

\section{Basic facts}

Among the targets the new units are aimed at, the essential one is keeping the adjusted temperature of air in the rooms and corridors of the building. The air is processed in the central plant. It may seem an obvious choice to transport the air to rooms by air flows in distribution ducts. Perhaps surprisingly, this choice is far from universally preferable. It is very expensive compared with heat transport by circulating water. The air flows require approximately $\sim$ 15-times higher power input and as much as $\sim 2$ 000-times higher volume flow rate (which means a correspondingly large internal space in the building occupied by the

*Corresponding author: tesar@it.cas.cz 
ductwork). This has led already in the earliest central heating systems to the use circulating water and the heat exchangers (called "radiators"- though the heat transfer is actually more by convection than radiation) inside the room. The historic not well controllable systems with free-convection air flows - still common in some part of the world - are not satisfactory. It is desirable to use instead the better controllable forced convection, with air jets issuing from suitably located terminal units containing the heat exchanger. In their present layouts, however, terminal units are quite expensive both to manufacture and to operate. There is, however, no universally best solution. It is necessary to accept compromises, dependent primarily on the magnitude of heat transfer rate. Analyses show that the water/air systems are preferable if the thermal loading of the room is high. The limit is at about $\sim 80 \mathrm{~W}$ per $\mathrm{m}^{2}$ of floor surface [5]. In thermally less loaded rooms the economy usually prefers an all-air arrangement, a typical example of which is shown in Fig. 1.

Apart from the heat transfer, a secondary but also necessary task fulfilled by the conditioning system is ventilation of the rooms, i.e. removal of contaminated air, irreparably polluted by exhaled humidity, $\mathrm{CO}_{2}$, odours, and aerosols - such as, e.g., cigarette smoke. The contamination level, however, is usually not really too high so that a return of some room air to the blower or to the air transport ducts is acceptable (and preferable economically). The essential reason for the removal of the air from the rooms into the atmosphere is the need to provide a space for the incoming fresh processed air, which must be delivered in the amount capable of achieving and maintaining in the rooms the required thermal equilibrium.

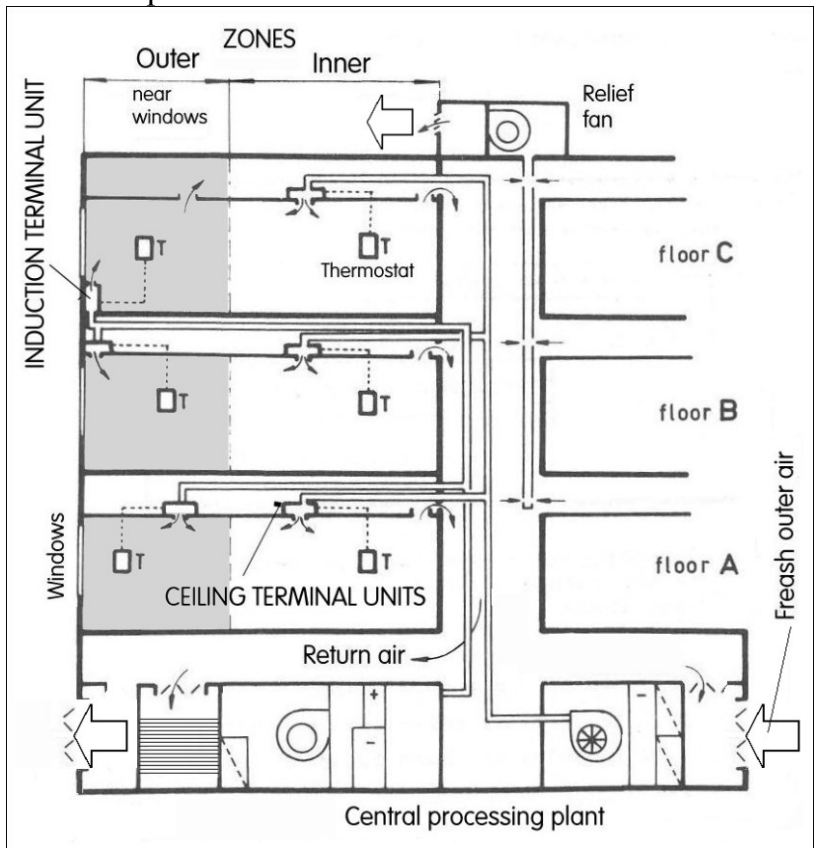

Fig. 1. An example of an all-air heat transport and its control in a building. Processed in the central plant is a mixture of fresh and returned air. Rooms are divided into zones (gray and white) with separately controlled air conditioning.

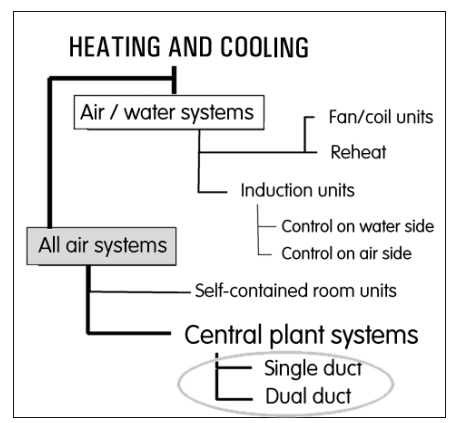

Fig. 2 Alternative principles of heat transport and control for comfort environment in buildings.

Of course, the removed air dumped into atmosphere carries away the thermal energy that was spent earlier on its heating. This may represent a significant energy loss, especially if the outer temperature in winter or northern latitudes is low. At least partly this loss can be in principle made up in heat exchangers. Currently it is seldom done because the available temperature differences are rather small so that the corresponding exchangers of the usual recuperation type [18] are too large and too expensive. It is also here where fluidics can offer an interesting solution. Because of its ability to switching the flow very fast without problems like the wear of mechanical components, it is possible to use for the purpose a pair of switched regeneration type exchangers of quite small size. The presence of the heat-exchanger matrix in the terminal unit can bring the additional advantage of suppressing the aerodynamic noise generated in the upstream parts of the system.

Of critical importance for room occupants' satisfaction with their heating system is the quality of control, especially the control of temperature [3]. The problem is the uncertainty and problematic criteria of the satisfaction. Experience shows that only $60-75 \%$ of persons (and in some documented cases very much less) are satisfied with the same temperature. Problems arise especially if there are in the same room many persons with non-uniform opinions. Physically less active persons demand significantly higher temperature while those more active demand a level much lower. The problem is aggravated by the large time lag of the control loop, which involves the large thermal capacity of the room The result is the occupants incessantly tampering with the thermostat setting and the consequent rather general dissatisfaction. Together with the objective fact of unequal thermal loading of spaces positioned differently in relation to the warm-air outlet, the target of general satisfaction simply calls for dividing the heated spaces into separately controlled zones, Fig. 1. Until recently it used to be common that a single control zone included several rooms having similar expected thermal loading. Contemporary trend is the other extreme, dividing even relatively small rooms into several zones with private air outlet for each occupant - if his position is stationary at least for some time. This refinement, of course, requires more terminal units. It also means more room temperature sensors. 


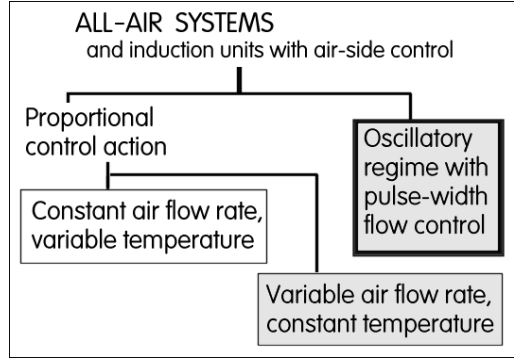

Fig. 3 The character of control by jet switching in fluidic valves prefers operating the fluidic terminal units in the oscillatory PWM mode regime.

This solution may be thus quite expensive in terms of the first cost. Nevertheless, it provides another opportunity for a compromise. The smaller controlled spaces may lead to significantly lower operating costs as there is no more the need (common earlier) to satisfy the most demanding room and the most demanding person. This was achieved at the price of overheating the zones and spaces occupied by less demanding inhabitants. Obviously, the trend of local comfort with many terminal units creates a pressure on low terminal units price - and this is where the solution can be found using the ideas taken over from fluidics. Apart from their low initial cost, the economy of fluidic units is aggravated by their long life and generally no need of maintenance.

\section{Systems configuration and control}

The transport of heat inside the building may be performed by many alternative system configurations. The illustrations Fig. 2 and 3 present the "taxonomic trees" of available possibilities. There is no universally best approach. A rough guide for the choice is the magnitude of thermal losses in the rooms. The losses are evaluated as the supplied thermal power (in watts) per length of the perimeter walls.

Of very old history, beginning already in 18th century, are the air/water systems. The idea originally started with heating of greenhouses. The first practical system built Perkins in London in 1832 for growing grapes in cold climate. It was based on the then new idea of screwed connections of metal pipes, withstanding the necessary pressure without leakage. In 1855 San Galli made another important step by inventing relatively inexpensive cast iron "radiator" heat exchangers. Requests for proper temperature distributions inside the heated rooms have led to today's air/water systems. The air is forced to flow past a heat exchanger in the terminal unit. It may be driven by a local blower but economy prefers air flow driven by central compressed air source and distribution throughout the building by a ductwork parallel to the water system.

There are locations inside many buildings - typical case are those near windows or doors leading outside where the local thermal losses are really high. The inhabitants there should be protected from the freeconvection streams and jets of cold outside air, especially in winter. Typically, the forced warm air jets are arranged to issue under the window sill, as shown in the $\mathrm{C}$ floor in Fig. 1. This is the best location for the relevant terminal units in situations with very high thermal losses, above $\sim 400 \mathrm{Wm}^{-1}$ (- the length scale is length of wall perimeter ).

Using the advantage of efficient heat transport by circulated water, present-day window-sill terminal units $[11,13]$ are now usually of induction, jet-pumping type, cf. Fig. 2. Their main task is suppression of discomfort caused by cold. For this purpose, the flow rate of air issuing from the exit of the unit is usually much larger than what is needed to remove the contaminated air. Thus it is generally quite acceptable to re-circulate the same room air past the heat exchanger several times.

For medium heat losses $\left(240\right.$ to $\left.385 \mathrm{Wm}^{-1}\right)$ there is a positive experience with an over-window terminal unit as shown in floor B of Fig. 1. If the magnitude of thermal loss is low (below $200 \mathrm{Wm}^{-1}$ ), a special nearwindow unit is not necessary (Fig. 1, floor A) and suitable positioned ceiling-type terminal units - same as for all other zones - are usually preferred on the grounds of cost savings achieved by the standardisation.

The requirements of ventilation (air exchange) are more demanding in multi-zone heating systems with low thermal losses. Their terminal units are usually located above the false ceilings of the rooms, the space co-occupied by supplied heated-air ducts and return-air ductwork. In the present-day all-air systems (cf. the tree diagram in Fig. 3) the standard control mode is proportional, with heat delivered in proportion to the demand. There are two approaches. An often used layout is the constant-flow approach [5], with air temperature being the controlled variable. Most often this has been implemented in the form of the dual-duct arrangement, ref. [7]. One of the two parallel ductwork systems conveys cleaned cold air while the other duct conveys the heated air. Seasonal conditions calling for only small-scale heating or cooling are handled by the central controller in the air processing plant switching one of the ducts off. Otherwise (in spring and autumn) both ducts are active and their air flows are mixed in the terminal unit in proportion to the local demand, while the combined air flow rate remains constant. Bringing up to the terminal units both hot and cold water may seem uneconomical, but is is justified by the rapidity of the response to changed demands.

The other approach to the all-air systems is the constant temperature version (cf. Fig. 3) with the terminal unit varying the air flow rate into their zone. Economy demands returning to the central plant the processed air prevented from entering the heated room. For this purpose the second ductwork has to be there anyway. One of obvious disadvantages is the variation of the flowfield inside the room as a consequence of the varied velocity in the exit from terminal unit. The slow air jet cannot reach the same horizontal distance as does the more powerful one at the higher exit velocity. Another obvious disadvantage is also the wasted energy spent on transporting the diverted air from the central plant up to the terminal unit. If this diverted air is mixed with the contaminated relief return air from rooms in an attempt to save a special return ductwork, then 
additional loss arises of the heat content of this discarded air (apart from the effort spent on its filtering). The advantage of constant flow through the central plant may seem insignificant at the first sight when compared with these losses. But the variable flow through the central unit with the throttling has a serious consequence: large thermal capacities prevent it from reacting sufficiently fast. As a result, there are situations of sudden changes of flow rate from many terminal units, which leads to large excursions of temperature of the supplied air. This, together with the accompanying unbalance of pressures in the ductwork system can make the target of a precise control quite elusive [5].

As might be expected from designers accustomed to the general earlier use of mechano/fluidic terminal units, initial applications of fluidic units were based on direct analogy. The trend was continuous air flows with varied velocity in the unit exit. Indeed, large-scale fluidic valves able to operate in this continuous control mode may be (and were) built. In contrast to the earlier mechanical versions where the movable component does not respond by motion (and flow rate change) responding to pressure distributions, the continuous fluidic versions without mechanical components were found very difficult to adjust for a particular application. The aerodynamic properties had to be set up and tuned to particular air supply sources and room conditions. Perhaps their more important negative factor was their disadvantage of requiring quite powerful incessant control input flow, for example to keep the jet deflected, cf. $\mathrm{A}$ and $\mathrm{C}$ in Fig. 4. The fluidic terminal units have to be operated and controlled differently. A particularly interesting solution is the use of switched Coanda-effect valves which during most of the time need no input control power at all - the jet stays deflected and the input control flow is needed as only a brief flow pulse for the switching.

\section{Flow control principles in pure fluidics}

In the "pure fluidics" generation and control of fluid flows are performed without engaging any mechanical movable or deformable component. The control effects is generated in constant-geometry cavities, using fluidmechanics phenomena such as hydrodynamic instability. The whole flowfield inside the device responds to a weak input flow. The absence of mechanical components means a fluidic flow control device is nothing more than body with suitable shaped internal cavity for the fluid flows. Admittedly this cavity may be of a rather complex geometry, but this is no problem in view of present-day availability of advanced manufacturing methods - especially the additive manufacturing - which can produce the cavity and all component parts in a single manufacturing step, avoiding expensive manual operations like assembly from separate components. There are no springs or membranes that might rupture, no bearings that may get stuck or need lubrication. There is also no need for electric components and this means no danger of shortcircuit fires.
Until recently, development in fluidics was focused on small size devices. It was due to the intended applications seen in small-scale and microscale devices and systems. [1] In fact, most present-day fluidic devices are still small and there is a trend of manufacturing them by the methods taken over from microelectronics (with which the fluidics may actually share the substrate). Typical character of small devices operation, similar to those in digital microelectronics, is discrete actions and bistability, alternating between two stable regimes. Typical problems that may arise with the small scale is the necessity to have Reynolds number of flow above a certain limiting value $\mathrm{Re} \approx$ 800 [2] necessary for reliable functioning of the Coanda effect [4] — the attachment of fluid jet to a nearby wall, in fluidics very often used. Fortunately, for the large-scale devices in the terminal units of the air conditioning systems there is no such physical limit.

In the simplest of approaches, the scaled-up fluidic devices can replace the present-date mechanical valves, dampers or similar devices with moving parts, retaining the same mode of operation. Even this simple replacement strategy can bring several advantages, such as the easy manufacturing, no maintenance, no wear and therefore long life, and last but not least the low cost. However, because of the absent inertia of components and no wear of contact surfaces, fluidics makes possible operation in regimes which mechanical parts would not allow at all or at which they would rapidly deteriorate. Typically, fluidic valves can operate in permanent ONOFF oscillation between two regimes, modulating the time-mean effect by varied duration of the $\mathrm{ON}$ pulses.
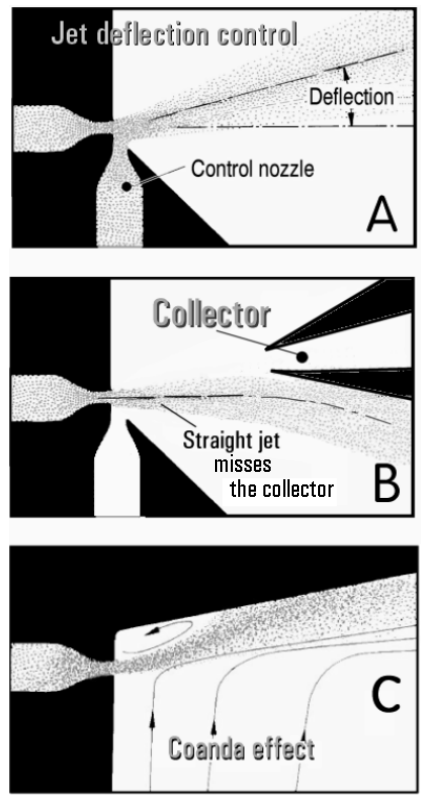

Fig. 4 The most important among fluidic no-moving-part flow control principles are deflection of jets. A - Jet is directed by momentum interaction with the flow coming from the control nozzle. An amplifier has a collector capturing more or less the deflected jet, leading it to the output terminal, C - Often used Coanda effect keeps the jet deflected by attachment to a wall even in absence of any control flow. 


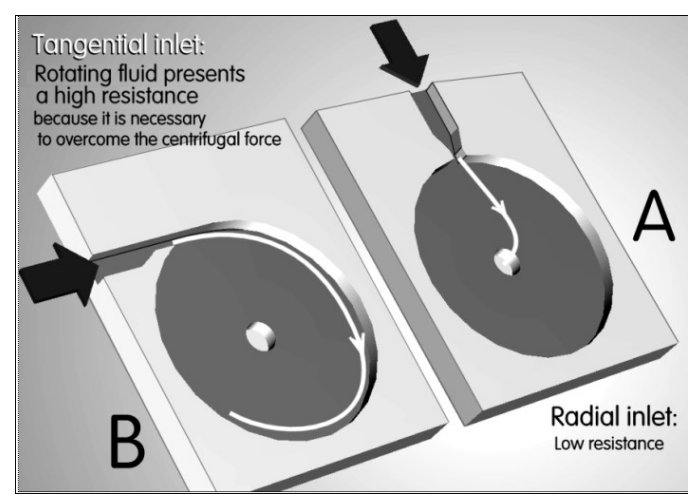

Fig. 5. Another control effect used in fluidics is the difference in resistances between radial non-rotating flow $A$ and the high-resistance tangential regime $\mathrm{B}$ in which the flow has to overcome the centrifugal acceleration.

This can actually bring several advantages specific to the room ventilation. Present-day air flow control in buildings uses mostly dampers turning-down the efflux. This means decreasing the air flow velocity when the demand for air jets lower. The unpleasant consequence of this decrease is the variation of the flowfield inside the room. The slower air flow, leaving the terminal unit with lower momentum, flows differently that a faster flow - especially if it is under the influence of hydrostatic lift force acting on the warm air jet. The fluidic solution with varying pulse durations but constant velocity during the flow pulse, can control the amount of air flow and heat it transports keeping invariant the room flow pattern, i.e. character of the flows in the room.

Another advantage of fluidic versions of the terminal units is the absence of electricity in the system. This makes superfluous the otherwise necessary and omnipresent conversion transducers - both the fluido/electric ones at the sensor side (in room thermostats) and the electro/fluidic ones for valves or blowers on the actuating side of the control system. In the fluidic version of the system the controlled air flow serves simultaneously for three purposes: as the heat carrier, working fluid in control signals generation, and for transport of signals between components. It may be even used for signal processing. It may be inexpensive and, as mentioned above, avoids the danger of fire caused e.g. by accidental short-circuiting. Particularly favourable is the fact that the air for the transfer and signal handling passes through the central processing plant where from it is removed dust and excessive humidity - the two worst enemies (causing clogging) of pneumatic systems at small scales.

Essential components of the discussed terminal units are fluidic valves controlling the air flows. In principle they operate as amplifiers, with a weak input air flow steering the large output. It is achieved by applying the input action in some sensitive spot of the flowfield. The most popular mechanism is the jet deflection principle presented in Fig. 4. The controlled main air flow forms a jet by issuing from a nozzle. Upon leaving its exit it is deflected, as shown in A of Fig.4. At the other side of the interaction cavity is the jet captured in a collector. The controlled deflection means ( $B$ in Fig. 4) variation of the flow 4, with the jet remaining deflected even if there is no control flow. Both principles $A$ and $C$ in Fig. 4 may be combined, the control flow deflecting the jet towards the attachment wall where it then remains even if the control flow ceases. An amplifier is set up from the configurations shown in Fig. 4 by addition of the collector capturing the jet fluid ( $B$ in Fig. 4) and delivering it to the amplifier output. With increasing jet deflection in the configuration B in Fig. 4 the air flow rate in the collector also increases. The ratio of the two flow rates (output in the collector to input in the control nozzle) is the flow gain $G$. Fluidic amplifiers do exist that reach the values as high as $G \sim 100$. However, even a lower value may suffice for a number of useful tasks. Of course, the Coanda-effect case C in Fig. 4 operates in only two output flow regimes, ON (with the jet attached) or OFF (jet separated). This is enough for the pulse-width modulation control mode - and may have the advantage of very low control flow time-mean consumption (the control action is needed only very briefly for switching the jet).

Another flow control principle in pure fluidics uses vortex effect in fluid that rotates in a cylindrical chamber, as presented in Fig. 5. If the fluid enters the vortex chamber tangentially (the case $A$ in Fig. 5), the fluid inside the chamber rotates. Because of its tendency to maintain the moment of momentum, the rotation speed increases as the fluid progresses towards the central exit. With it increases also the centrifugal force on the rotating fluid. This may become so high that getting to the exit is very difficult. In theory the centrifugal force may decrease the flow rate nearly to zero - with only some small flows passing through the boundary layers on chamber floor and ceiling, where the rotation speed is lower due to wall friction. On the other hand, in the regime $B$ in Fig. 5, with radial entry into the chamber, the hydrodynamic resistance of the whole device may be quite low.

Number of known fluidic amplifier principles and configurations is large and some of them are little known. An example of an almost unknown case tested by the author is the very special valve presented in Fig. 6 . The normally straight flow is deflected by control flow applied to the slits at the trailing edges of parallel vanes

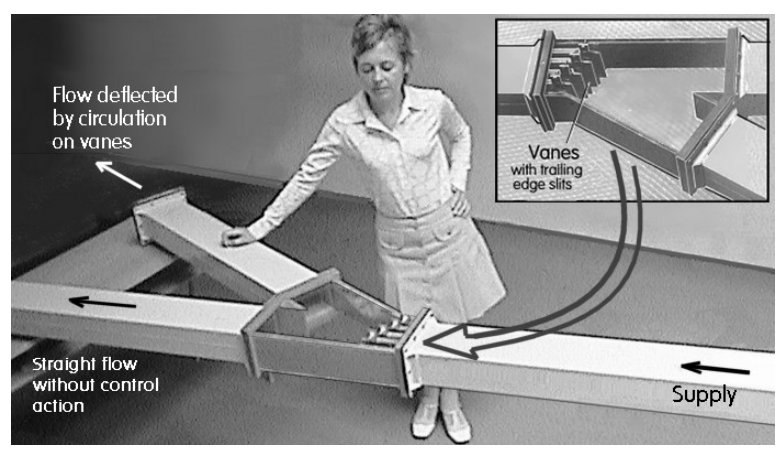

Fig. 6. Fluidic diverter valve in the $100 \mathrm{~mm} \times 150 \mathrm{~mm}$ laboratory-tests rectangular section ducts. The unusual method of flow deflection tested in this case was the circulation on fixed (non-movable) four parallel vanes with control jets attaching by Coanda-effect to vane trailing edges. 


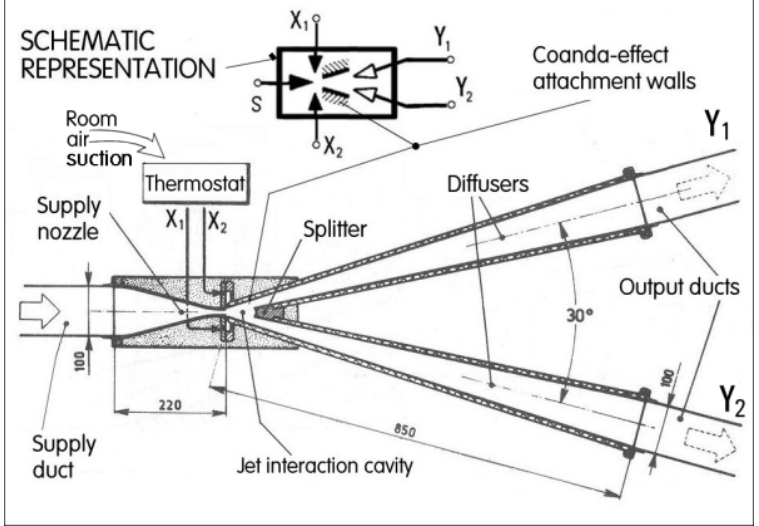

Fig. 7 Bistable diverter valve based on the jet deflection and Coanda-effect attachment. This test model was used in terminal unit off $100 \mathrm{~mm}$ wide rectangular model ducts. The area contraction in the supply nozzle together with the pressure rise in the long diffusers creates in the control pipes $\mathrm{X}_{1}$ and $\mathrm{X}_{2}$ a sub-atmospheric pressure, which is used to suck into the valve the room air passing en route through the thermostat.

In contrast to the movable vanes in Venetian blinds, here the vanes are fixed, not changing their attack angle. They are hollow with the control flow passing through their internal cavities, leaving them through the slits at the trailing edges. At these edges are also small Coandaeffect attachment walls causing the jet inclination. This generates on the vanes a circulation and consequent deflection of the main flow - essentially the same circulation as would be obtained with inclined vanes.

An example of the currently most important type of fluidic valves is presented in the next Fig. 7. It is a drawing (and schematic representation) of a model used in laboratory tests of fluidic terminal unit for model size $150 \times 100 \mathrm{~mm}$ rectangular cross-section ducts. The valve is bistable and its geometry is fully symmetric. In the schematic representation used in circuit diagrams, shown here in the top of Fig. 7, the filled (black) triangles are symbols for the nozzles - the supply nozzle and two control nozzles $X_{1}$ and $X_{2}$. Empty (white) triangles represent two exit diffusers. Also presented in this schematic representation are the two symbols for attachment walls, like the wall in the case $\mathrm{C}$ of Fig. 4, now in a pair with one wall on each side of the jet issuing from the supply nozzle. It is this wall pair that makes the valve bistable, i.e. able to stay without control flow in one of two deflection regimes. Air flow enters the valve through the supply nozzle of $150 \times 7$ $\mathrm{mm}$ exit size. The control nozzles are thin $150 \times 2 \mathrm{~mm}$ slots. The valve was tested with several alternative splitter shapes opposite to the supply nozzle. The best results were obtained with the bicuspid geometry having the splitter nose with concave wall between the two cusps. After attachment to one of the two attachment walls the flow is captured by $150 \times 14 \mathrm{~mm}$ (i.e. 2 nozzle widths) collector entrance and then led through long, 6 deg angle diffuser in which the kinetic energy of the flow is converted back into the pressure energy. This generates a sub-atmospheric pressure in the interaction cavity as well as in the two control flow pipes leading to $X_{1}$ and $X_{2}$. The control - by switching the jet between the attachment walls by opening the control inlet into the atmosphere - thus does not need any external blower.

\section{Simple flow switching: Fluidic water/air induction units}

\subsection{Symmetric fluidic terminal unit with neutral central channel}

Induction type terminal units [11] are typically used to cover local high thermal needs, typically next to windows. They often operate with active both hot and cold water supply to adjust the temperature of generated air flow. In principle, of course, it is possible to use only one water circuit (hot in winter or cold in summer), but this may result in unpleasantly slow response to a change in temperature requirements. Recirculation of the room air is standard because the delivered thermal input does not correspond to the much smaller demands of contaminated air replacement The new fluidic units are nowadays starting to supersede the earlier convection units electrically driven by a local blower, usually suffering from noise and rather high cost of electricity consumption.

Modern approach is supplying the pressure air from the more economically operating central plant where the air, in addition to its pre-heating (or precooling, dependent on the season) is also filtered. Using the jet-pumping effect, this air from the central plant drives the air entrained from the room. The example described in [10] of the fluidic version of the induction terminal unit is here presented in Figs. 8 and 9. Its internal layout strongly resembles that of the bistable diverter valve from Fig. 7. The compressed air from the central plant generates the primary jet by flow through the supply tube at the bottom of the unit, leaving this tube through the slit-shaped primary nozzle. By the jetpumping suction effect is entrained recirculating air from the room. It enters through the filtering textile screens

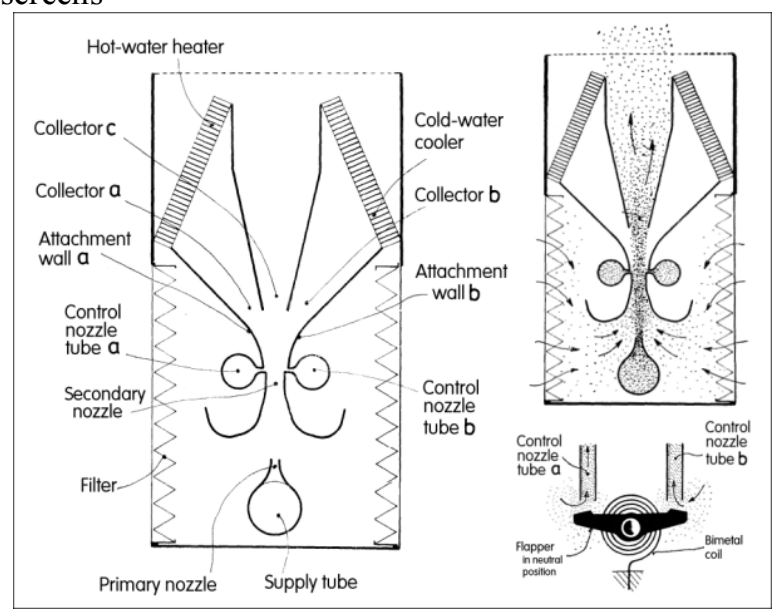

Fig. 8. Application of the Coanda jet attachment flow diverter principle in the water/air terminal unit [10] of induction type, driven by supplied clean air from central plant. At right is the bimetallic thermostat in the neutral, central jet position and the corresponding non-deflected straight jet flow. 
on both sides of the body of this terminal unit. Their task is to remove from the air the aerosols, such us the dust carried into the room on inhabitants' shoes. Of course, this causes the disadvantage of needing a periodic filter maintenance, but without this filtering the dust would re-circulate in the room permanently. The mixture of the primary and recirculating air then enters the secondary nozzle. This generates a wider and slower secondary jet, moving upwards through the space divided by sheet-metal

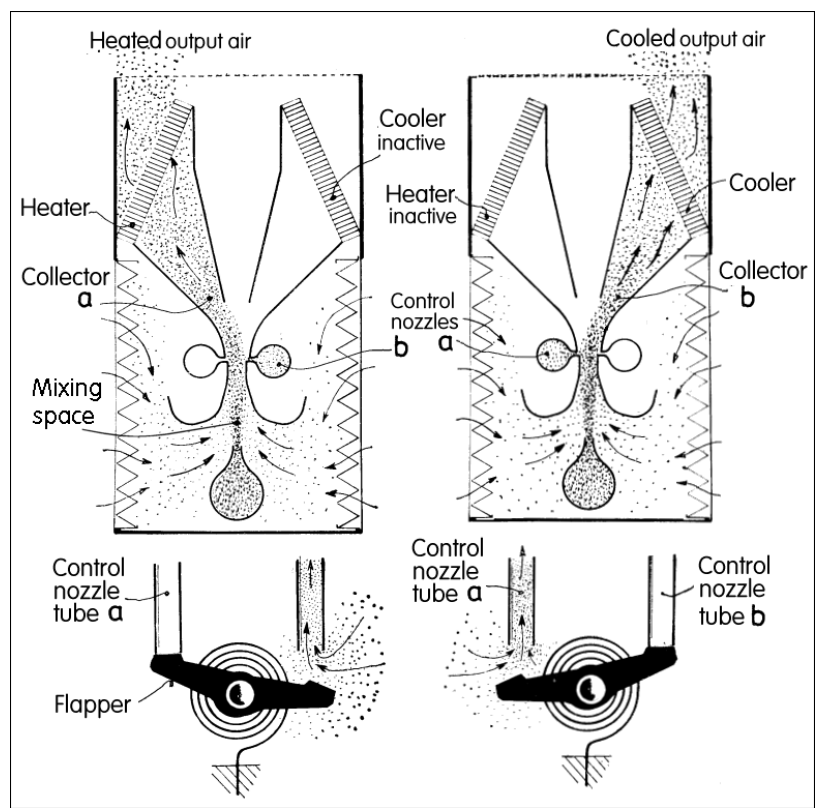

Fig. 9. Two flow regimes in the window-sill terminal unit shown in Fig. 8. The air jet is switched by closing the suction from the atmosphere by the bimetallic flapper thermostat.

partitions into three collectors. The collector $\underline{a}$ at lefthand side leads the secondary jet, as shown at left in Fig. 9, to the heater (with hot water circulating in it). When the jet leaving the secondary nozzle is switched to the opposite side, it passes through the second collector $\underline{b}$. This leads - as shown in the right-hand side of Fig. 9 - into the cooler (containing cold water). The bottom part of Figs. 8 and 9 shows how the switching between the jet flowpaths may be simply achieved by opening or closing the room-air suction into to control signal tubes connected to the control nozzles $\underline{a}$ and $\underline{b}$. The pair of small-diameter tubes connect the flapper sensor with the terminal unit. The flapper is two-armed, positioned at some distance from the unit, preferably at the wall of the room, above the room floor at the level of human face. The arms of the flapper are inclined by the spiral bi-metallic coil responding to changes in room temperature. If the temperature is in agreement with the desirable level, the flapper (as shown at righthand part of Fig. 8) is in the middle position. In this regime the air enters solely for the ventilation purpose, jet deflection eliminated due to suction into both control tubes ( $\underline{a}$ as well as $\underline{b})$.

\subsection{Induction terminal unit driven by jet- pumping suction at the downstream end}

Another variant of fluidic bistable induction-type terminal unit as originally discussed in [10] is presented in the next Fig. 10. Also here the overall flow is directed upwards from the bottom of the unit, where enters the re-circulating room air. Configuration resembles Fig. 8 but here is no middle collector - and the flow through the unit is driven by the primary jet of pressure air (delivered from the central plant) at the top of the unit. There are several alternatives possible of the layout of the jet-pump effect. Fig. 10 shows both alternatives at the same time (only one of them is, of course, sufficient). In parallel with the nozzle shaped as a slit in the horizontal supply tube, is shown the Coanda-effect nozzle with the jet initially following the curved outer wall of the supply pipe to which the nozzle is oriented tangentially.

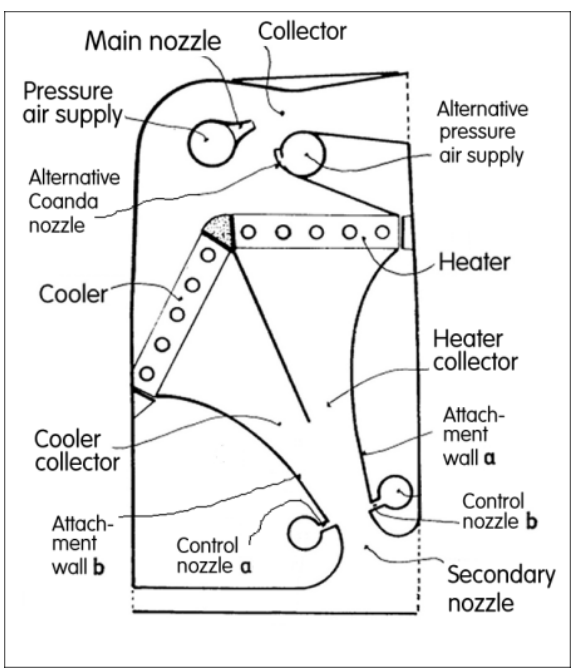

Fig. 10. Alternative induction-type fluidic jet-attachment diverter [7] in the terminal unit of water/air window-sill terminal unit.

\section{Pulse-width modulated oscillation}

\subsection{The PWM control}

Fluidic diverter valves, the main parts of terminal units controlled by small flow rate input signals are, in principle, fluidic amplifiers. In analogy to electronic oscillators, which use the feed-back effect together with phase-shifted amplification, the fluidic oscillators are similarly set up. In the oscillator role, the amplifiers are provided with a feedback loop flow channel, connecting the control terminal with some other terminal in which the increasing control flow decreases the output. Typical effect employed is the jet deflection and another 


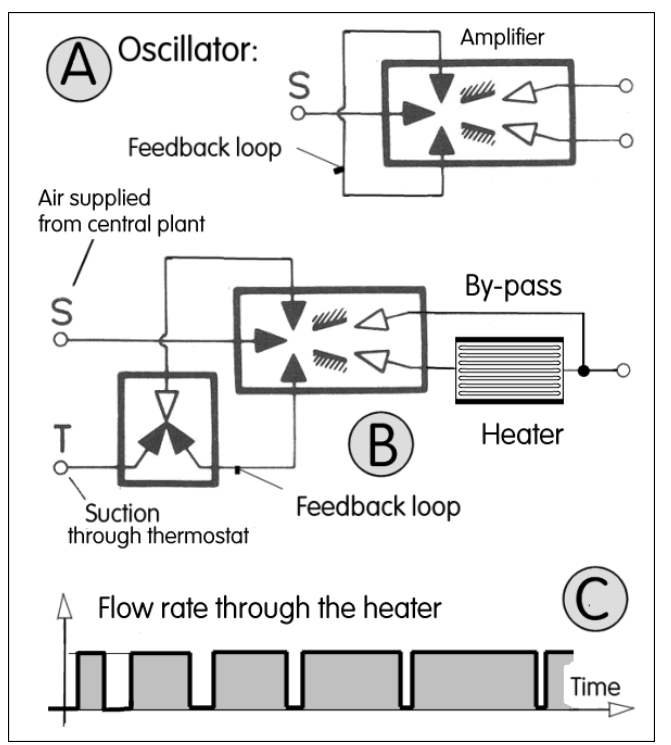

Fig. 11. Schematic representation of typical fluidic oscillator $A$, and of heater flow control $B$ by the input flow $T$ through the jet pump placed here into the feedback loop. The diagram $\mathrm{C}$ shows the resultant pulse-width modulated heater flow.

property of a jet: its entrainment suction of outside fluid. This suction is the mechanism behind the flows in Figs. 7 or 9 through the thermostat inlets, $X_{1}$ or $X_{2}$. When both control inlets are connected, as shown in the schematic diagram $A$ in Fig. 11, The pressure at one end of the feedback loop is lower than the opposite one. Lower pressure is in the control nozzle on the $\mathrm{ON}$ side the side towards which the jet is deflected. The difference creates in the feedback loop channel a flow from OFF towards the $\mathrm{ON}$ side control nozzle. Intensity of this flow increases, after some time, to a degree which it switches the jet to the opposite side. There the same series of phenomena takes place, with the control terminals having exchanged their roles. There is another switching and this is continued as the Warren's [14] oscillation - a series of flow pulses..

This oscillation may be controlled. Of particular importance in the present context is fluidic control (modulation) of the widths of generated flow pulses. Shown in the circuit diagram B in Fig. 11 is the oscillator delivering controlled hot air flow. The heater is connected to one of the amplifier output terminals. How large percentage of the air passing through the oscillator is heated - while the remaining flow remains cold - depends on the controllable asymmetry of the feedback loop. Even if the loop were symmetric (as the one shown in A, Fig. 11), the heater causes asymmetry of oscillator output loading. This inequality of oscillation phases may be increased or decreased by the three-terminal jet pump connected into the loop, shown as $B$ in Fig. 11. Because of the jet-pumping suction into the jet and efficient diffusers both control nozzles of the amplifier are at low, sub-atmospheric pressure. This drives atmospheric air through the thermostat terminal $\mathrm{T}$ into the loop. There it accelerates the flow in one direction of the feedback loop flow while opposing it the other direction. This way the duration of control flow pulses is increased with increased flow through $\mathrm{T}$ - while decreasing the duration of the interval between the pulses. Shown in the bottom part $C$ of Fig. 11 is the resultant time dependence of the flow through the heater. The higher is the flow in $\mathrm{T}$, the longer remains the jet in the amplifier attached to the side that has its output terminal connected to the heater.

The next Fig. 12 presents in a circuit diagram [6] a similar fluidically controlled time-mean heating of the air flow available in the exit terminal, which is shown at the bottom of the picture. There is again a bistable Coanda-effect amplifier (at top of the picture) with heat exchanger connected to one of its output terminals. Instead of the Warren's feedback loop of Fig. 11 (connection of both control terminals), there is the more obvious feedback connecting the amplifier outputs with the same-side control terminals. There are actually two loops, on left-hand and right-hand side. The accumulation chambers A and B (empty spaces) in the loops are filled through resistors $\underline{1}$ and 2 . This decreases the oscillation frequency - the feedback flows have to wait for the chamber pressure increasing to the switching level. The control of the oscillation asymmetry is in this case achieved by the vortex amplifier in the left-hand feedback loop. It is a cylindrical chamber with two inlets, a radial and a tangential one. Presence of the vortex amplifier in the left-hand A side loop is balanced by the simple resistor 3 in the opposite right-hand B side. The tangential inlet of the vortex amplifier is connected to the temperature sensor into which enters air from the heated space (room). If the thermostat closes the air inflow from the room, the vortex amplifier remains in the non-rotating OPEN state, with easy passage of the flow in the lefthand loop. The higher is the flow through the thermostat, the more the air in the vortex chamber rotates - and the longer its takes to the feedback loop to fill the accumulation chamber A because of the need to overcome the centrifugal force. As a result, the flow in the amplifier output spends more time by flowing through the heat exchanger.

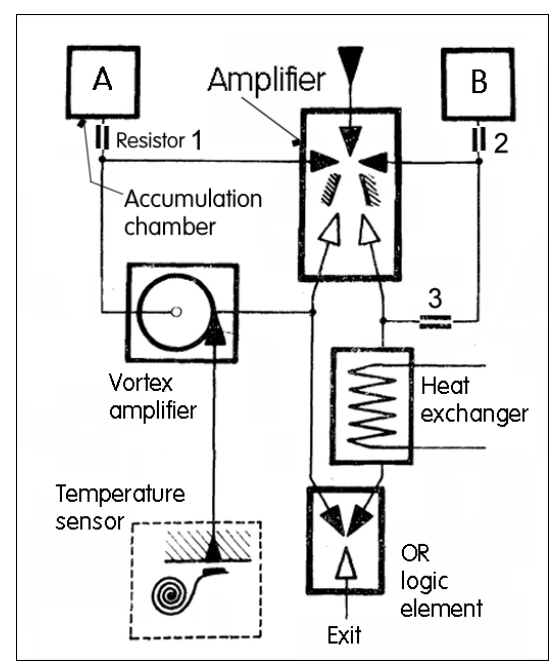

Fig. 12. Schematic representation of pulse-width modulation of heating the air in the terminal unit - with feedback loop asymmetry [6] controlled by vortex amplifier. 


\subsection{Monostable water/air unit with PWM control}

There are situations in which the induction type terminal unit has to generate only a hot air flow or cold one, but not both. Typically, this is found in modern buildings built from prefabricates - centrally produced and transported to the construction site. The transport aspects prefer the pre-fabricates to be light - which results in

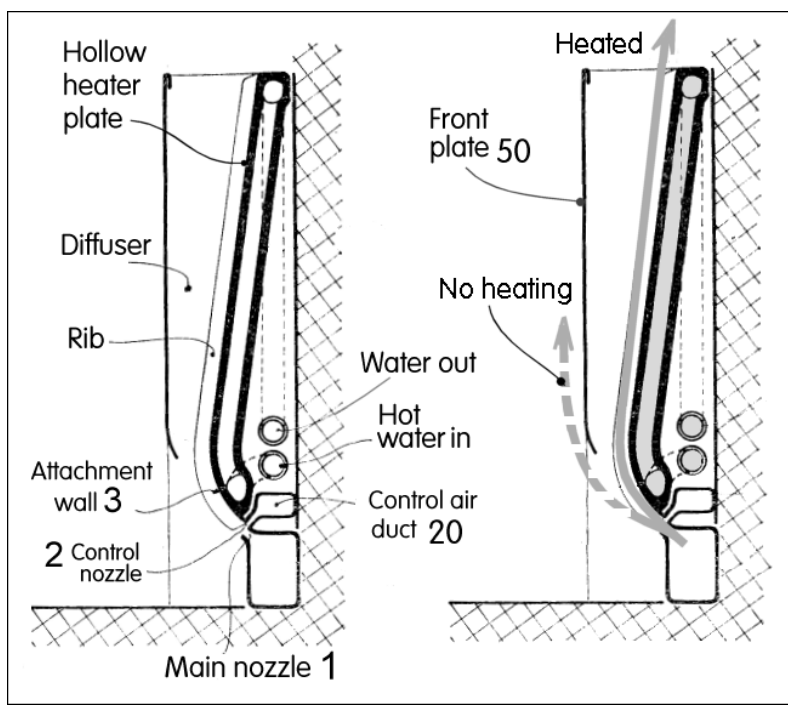

Fig. 13. Induction type window-sill terminal unit with monostable attachment of the jet to the surface of the hollow heater plate (provided with ribs). Numbers refer to components in Figs. 14 and 15.

their limited thermal insulation properties. The response of such rooms to changes in heat input may be in that case fast enough for needing only a single watercirculating system (only heating in winter or only cooling in summer).

An example of terminal units for this heating only task in a pure fluidics version is described in [8]. Here this terminal unit is presented in Fig. 13 in transversal cross sections. At left are indicated individual components while at the right hand side are shown by gray arrows the two operating regimes between the air flows are switched. It is either heating the room or ventilating the room without heating it. The next Fig. 14 shows the design of pulse-width controller and finally Fig. 15 is the circuit diagram of this whole unit. As typical for induction units, the heat is brought in by hot water. It flows in horizontal pipe of round cross-section. Flow of air in the room is driven by pressure air, delivered together with the water by ducts from the central plant. Available heat not used in this particular unit is removed by another, also horizontally oriented pipe of rectangular cross section. At the bottom of Fig 13 is this air distribution duct (10) provided with the slit of the main nozzle (1). The air leaving it forms a plane jet flowing past the control nozzle (2) and then offered the oppotunity to attach to a flat-plate heat exchanger attachment wall (3). This wall is provided with vertical ribs increasing the heat transfer surface and also securing two-dimensionality of the flow. The wall is slightly curved in its bottom part, this curvature promoting room air entrainment into the hot jet. The upper part of the attachment wall is straight and inclined, so that between it and the vertical cover plate (50) is a diffuser gradually increasing in width in the flow direction. In contrast to the bistable devices shown above in Figs. 8, 9, and 10, here the valve behaviour is monostable. In the no-control-flow regime the air jet remains stably attached to the attachment wall (3). In the response to the air control flow in the smaller rectangular control air duct (20), leaving it through the slit-shaped control nozzle (2), the main flow jet is blown away from the heater plate as well from the front plate (50). This means loosing contact with the heating. The monostability means the main jet has to be kept detached by a constant control air flow in the control air duct (20).

The controller part of the unit is at the air entrance side. It collaborates with a distant fluidic thermostat on the room wall, connected through two small-diameter pipes again using the low pressure inside the controller to bring in the controller a sample of room air. The main parts of the controller are in the five rectangular plates of different thickness - containing the air flow cavities in the main plate (110) and the central plate (120). Both are mutually separated by the thin partition (130). In the model for laboratory tests shown here the stack of these plates was held together by screws for easy access and changes. In bottom part of the stack all plates have a rectangular widow (11) allowing the air to pass through them to other terminal units further downstream. In the main plate (110) is in the top of the window (11) a primary nozzle (12) leading to the primary vortex chamber (14) through the pre-chamber (13). There it attaches to one of the pair of inclined primary attachment walls. Flow past one of them generates in the primary vortex chamber a radial flow while the other one generates a tangential direction inflow with consequent rotation. The air jet from the primary nozzle (12) is switched between these walls by alternating air flows in the Warren type feedback loop (29), connecting the nozzles (15) and (16). The flow in the controller oscillates in alternating flow direction in

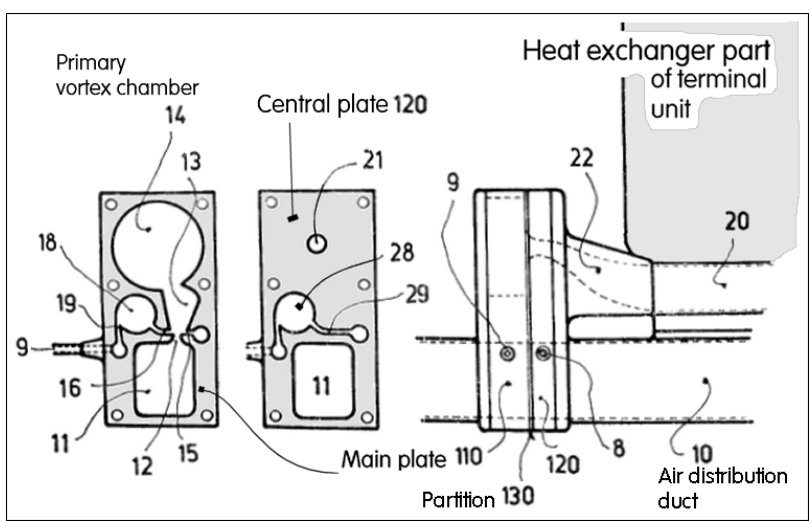

Fig. 14. Fluidic pulse-width controller of the oscillating-flow induction terminal unit shown in Fig. 13. Principle of operation is recognisable from the circuit diagram in Fig. 15. the feedback loop (29). In one regime the main vortex chamber (14) is in the OPEN state and flows through the 
exit (22) into the control air duct (20). In the other regime the flow into the duct (20) is minimised by centrifugal force. In contrast to the single vortex amplifier in the loop seen in Fig. 12, here as seen in Fig. 15 the loop flow modulation is done by two vortex amplifiers $(18,28)$ arranged back-to-back. Rotation in one of them modulates the width of the ON pulses while the other modulates the CLOSED regime durations between the pulses. Air flow from thermostat enters the controller through the terminals $(8,9)$.

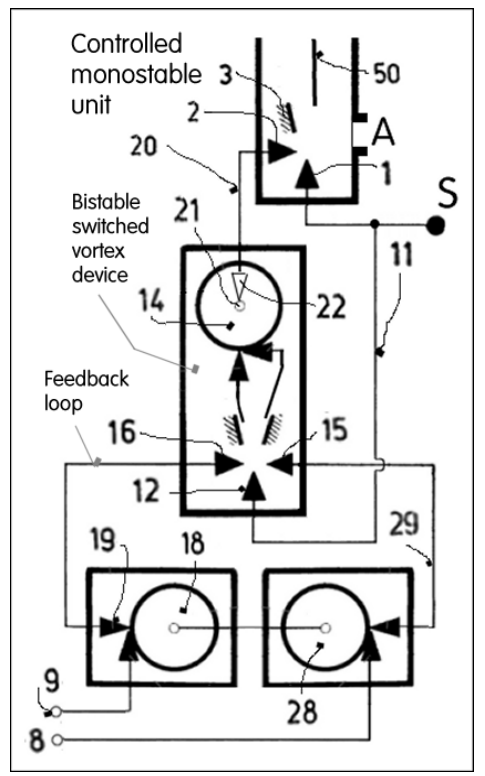

Fig. 15. Fluidic circuit diagram of the PWM controller for the terminal unit shown in Figs 13 and 14. The central device, Coanda-switched vortex amplifier, operates on the principle presented in Fig. 2C. Numbers correspond to [9] and to Figs. 13 and 14.

\section{System configurations - with the fluidic all-air oscillating terminal units}

\subsection{Terminal units with diverter valves}

The pure fluidic solutions are particularly suitable for buildings with thermally less loaded rooms (such as those conforming with the contemporary call for energy saving). They may operate in the all-air configuration, i.e. without transport by water from the central processing plant. In the version with diverter valves similar to the valve shown above in Fig. 7 - such a system is schematically represented in Fig. 16. The main problem caused by the use of the diverter-type valves as the key components in the terminal units is obvious. It is the loss of the energy spent uselessly on heating and transporting that part of the air which the terminal units prevent entering the rooms. Economic analyses show this type of terminal units is acceptable for buildings with of heat loss below $200 \mathrm{Wm}^{-1}$ built in mild climates. This is because the air flow rate into the rooms may be rather small and thus the absolute magnitude of the loss is acceptable. The decision to use this system configuration also depends very much on the controllability of the whole system by temperature demand adjustments applied in the central processing plant. The room thermostats then perform only small local changes.

The processed air not used has to be returned into the central plant. Thus an also non-negligible negative factor of this idea presented in Fig. 16 is the necessity of assigning a quite significant space inside the building to be occupied by the return-air ductwork. The first-cost economy may be somewhat improved, as seen in Fig. 16 , by combining the return flow spaces and ducts (serving for removal of the contaminated room air back to locations upstream from the filters. Paid for this is inevitable higher operating cost.

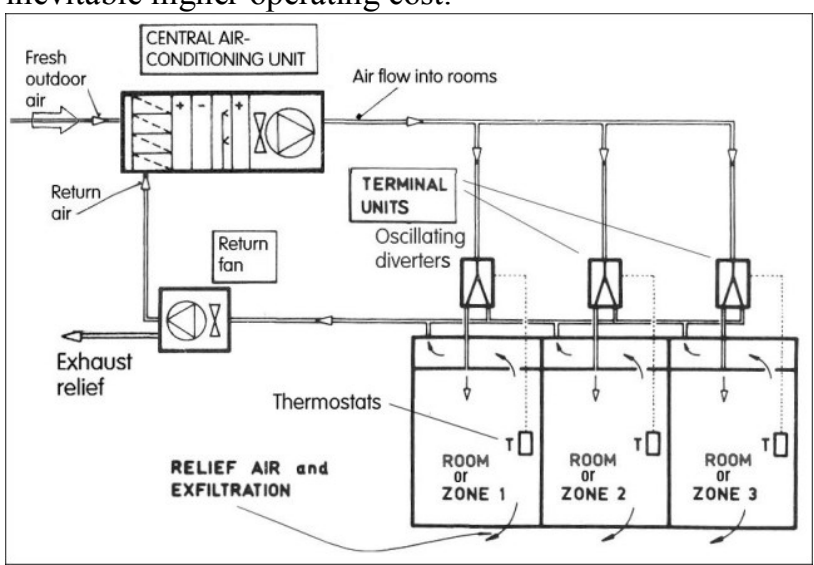

Fig. 16. Schematic representation of an all-air temperature control system with oscillating bistable diverter valves in the terminal units, operating in variable air-flow pulse-width mode. The ducts for diverted air are here combined with the ductwork for return air from rooms.

\subsection{Terminal units with bistable turn-down valves}

The problems with the diverted fresh air may be evaded by replacing the diverting type of valves in the terminal units by turn-down ones. The turn-down effect in pure fluidics is achieved by using vortex amplifiers. Schematic representation of the air conditioning system with oscillating pulse-width control of turn-down terminal units is in Fig. 17. In attempts at other applications of vortex valves there is a problem caused by their inability to turn down the flow completely, to zero. This has as been actually one of the main reasons why fluidics paid much more attention turned to the diverter valve types. Fortunately, in the building heating applications here discussed this inability is of no importance, since some small air flows into the rooms must be accepted anyway to pass through the valve for the purposes of ventilation. This inflow must be actually made possible by relieving some air from the rooms. This contaminated air may be simply allowed to escape into the atmosphere, but this is usually not economically acceptable - apart perhaps in cases like some hospital rooms generating a really dangerous contamination. It is preferable to transport the air back into the central plant which then does not need to heat the fresh air so much. Of course, providing the ductwork for the relief air, as shown in Fig 17, 


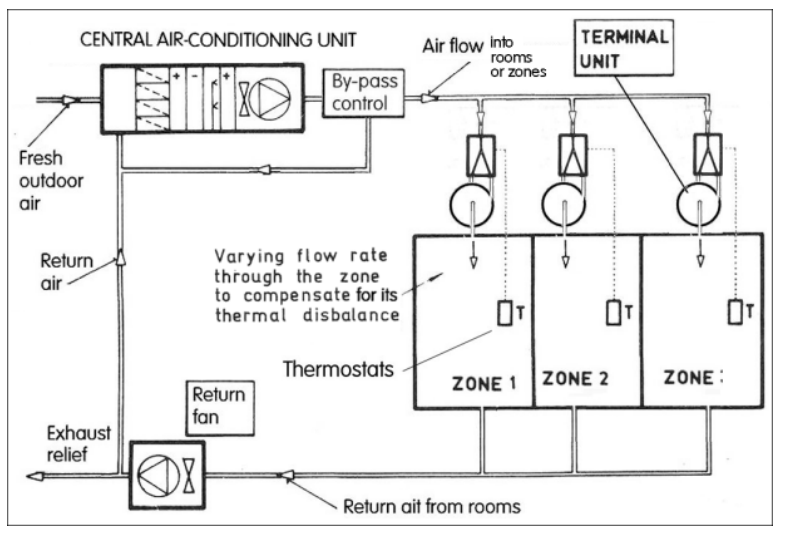

Fig. 17. Representation of thermal control in a building with all-air system using oscillating bistable turn-down vortex valves in terminal units operating in variable air-flow pulse width mode.- compare with Fig. 16.

means that there is not very much gained compared with the case of diverters in Fig. 16.

It may be suggested that there is a problem of the (admittedly rare) situation of all rooms in a building demanding the instantaneous CLOSED state in their terminal units. In fact the consequent blower stall is easily avoided with the by-pass as shown in Fig. 17.

\section{Bistable turn-down valve}

Inherent properties of fluidic flow control together with required properties of the fluidic devices in most applications prefer the diverting type valves. Somewhat exceptionally, the role in systems of the sort presented in Fig. 17 called for the turn-down operation, shown in Fig. 5. At the same time, it was preferable to retain the pulse-width modulation mode of control. The combination of both properties was achieved in the author's combination - otherwise little known - of a diverter with the vortex chamber. A laboratory test setup is shown in Figs. 18 and 19. The diverter valve was taken over unchanged from the design shown in Fig. 7 ad added was the vortex chamber. This approach

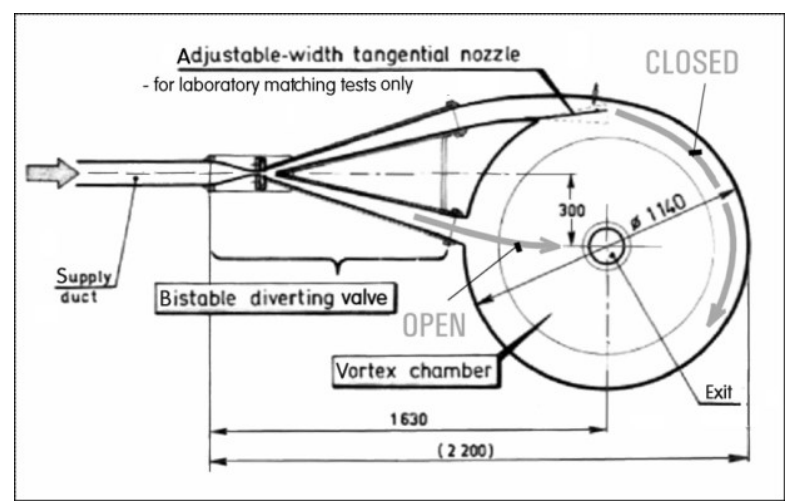

Fig. 18. The diverter from Fig. 7 was converted into a bistable OPEN/CLOSED valve. by attached vortex chamber. Advantage of this turn-down valve design is use of an previously well developed diverter part with known properties.

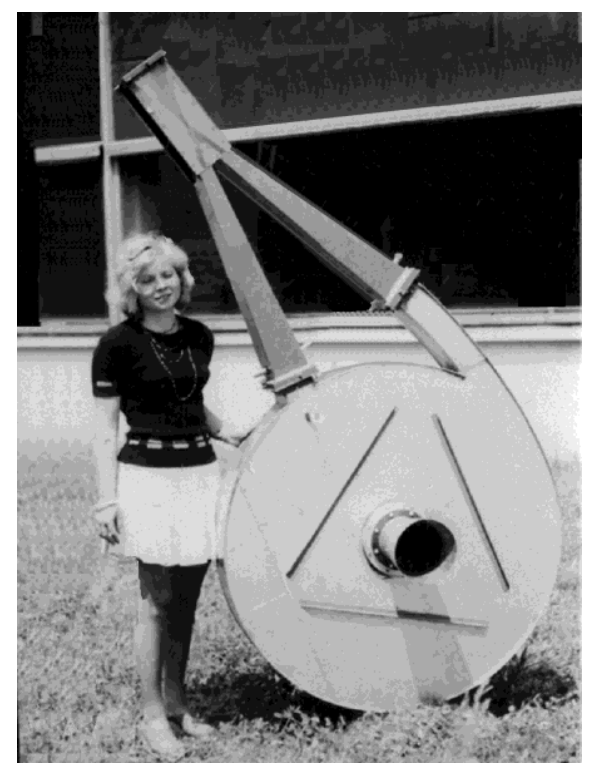

Fig. 19. Photograph of the bistable turn-down model valve made according to Fig. 18. The large size is necessary to keep low the velocities at which would be generated aerodynamic noise.

brings the benefit of standardisation, since both versions, for Fig. 16 and 17, use this common essential component. The diverter amplifier part is switched between two regimes. In one of them, air enters the vortex chamber radially, without large hydrodynamic resistance. When the diverter flow is switched into the other, tangentially directed terminal, the air in the chamber rotates. The centrifugal force significantly increases the fluidic resistance, generating the space between the output flow pulses. The large size seen in Fig. 19 is necessary to avoid aerodynamic noise [12] which is produced in smaller vortex chambers at higher velocities in. The adjustable nozzle for the tangential flow, seen in Fig. 18, was used solely for the laboratory optimisation tests. After the optimum was found, the flap was fixed.

\section{Fluidics applied to keeping inside the heat from removed air}

Ventilation - bringing fresh air into the living rooms - is a necessity. It is, of course, also make necessary to provide for it a space in the ventilated room by removal of the contaminated air. These flows out removes from the building a significant heat, on the generation of which was spent considerable energy. Standard approach in present buildings is to return into the central processing unit a percentage of removed air (usually in the vicinity of $\sim 20 \%$ of the total assuming it is note overly contaminated, the rest is left to escape into atmosphere). Apart from the drawback of recirculating the contaminants, this re-circulation 


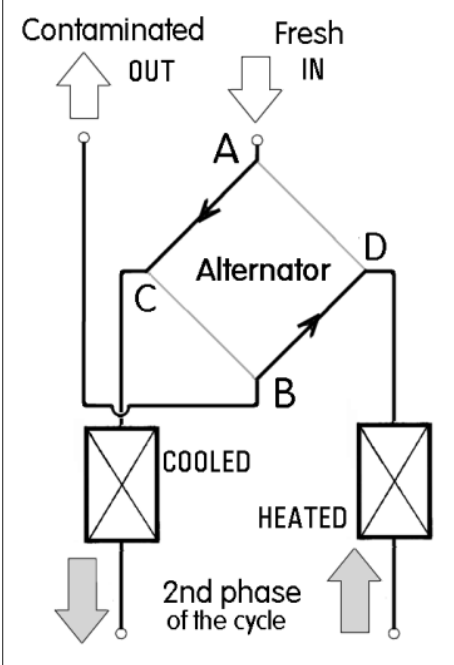

Fig. 20. Topology of the pair of regenerator exchangers with the air flow switched by a fluidic two-phase alternator [21]. Fresh air flows through A.

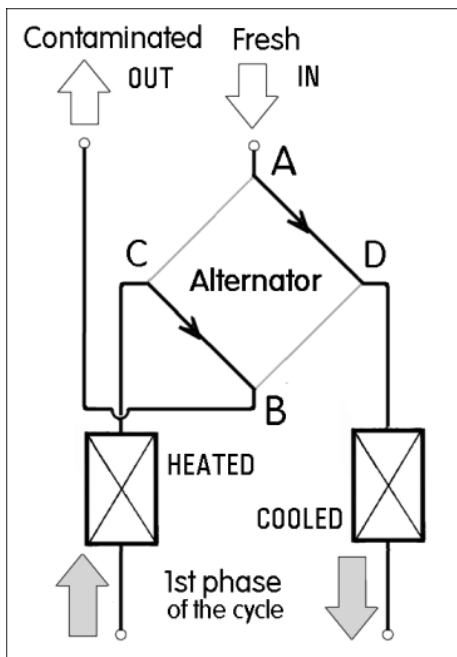

Fig. 21. Air flows in the other phase of the alternator than in Fig, 20. The fresh air here now flows through B.

approach has the disadvantage of requiring a considerable (and quite expensive) space inside the building for the return-air ductwork. Another handicap is the required power input - because the return flow is usually driven by a blower. An obvious possibility to the contamination problem is inserting between the two flows (fresh and returned air) a heat exchanger. However, ordinary recuperative type [18] heat exchangers, with two flowpaths inside its body separated by a thermally conductive partition, are for the air conditioning practically out of question. The temperature differences between the two flows are quite small. This would lead to a very large exchanger size and therefore too high investment initial costs.

A solution can provide heat exchangers of the different, regenerative type. There is through their internal matrix just a single internal flowpath and the flows of clean and contaminated air alternate in passing through it. If economically acceptable, the regenerative exchangers should be used in pairs to eliminate the waiting times between the thermal charging periods. Such two-phase heat exchanger pairs have been known in mechano/fluidic versions, switching the flows by mechanical valves. The exchanger may be small if the switching frequency is increased - but the rapid movements of the mechanical components acting on the air flows in succession generates noise and the valves call for driving electric power. Note in the topological schematic presentation of the two-phase configuration in Fig. 20 that the switching in principle needs four valves (in the vertices A, B, C, and D). Also a problem, the valves tend to deteriorate rapidly.

There is a possible but rarely used alternative operating without the valves by arranging the heat storage matrix in the form of a large slowly rotating wheel. The rotation alternatively places the inlets and outlets to the clean and contaminated air flowpaths. Like all solutions with moving components, this is not without problems. In particular, a reliable sealing of the inlets into the matrix wheel and out from it is a difficult task and the seals tend to be rapidly worn. The electricity spent on driving the wheel is expensive, as are the gears necessary for the reducing the rotation speed of the driving motor. The result is not only high first cost but also a non-negligible operation cost.

Pure fluidics discussed here offers a solution with no moving or deformed components, no seals and no motors. There is, as a result, no wear and no maintenance (replacing worn seals). The principle of operation in two alternative phases is presented in Figs. 20 and 21 showing the character of the flows in the two

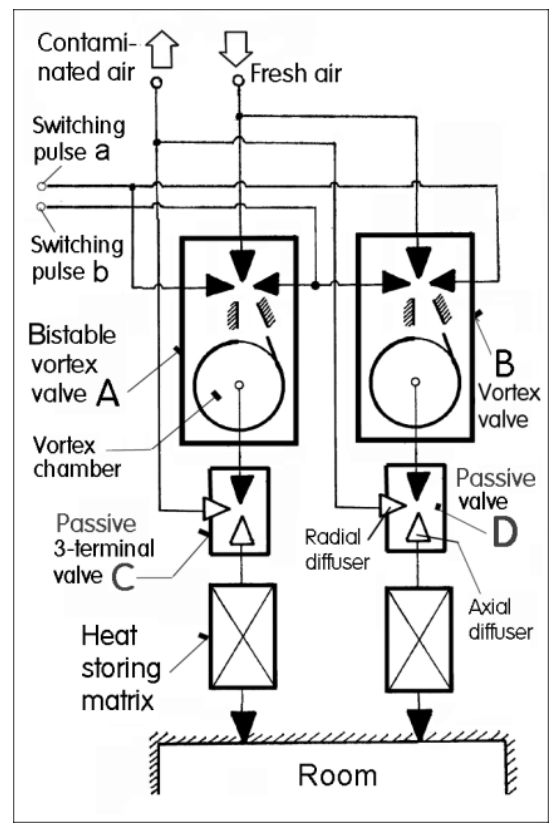

Fig. 22. Circuit diagram of an example of fluidic system recovering heat from the contaminated air [16] returned from the room and (at right) representation of the two air flows. Main components in this particular alternator are two bistable vortex valves Fig. 18. 


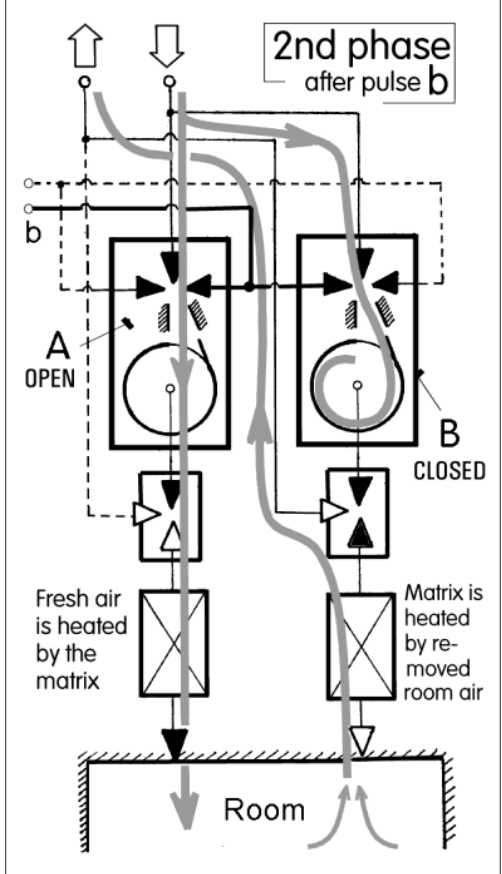

Fig. 23. Circuit diagram of an example of fluidic system recovering heat from the contaminated air [16] returned from the room and (at right) representation of the two air flows. Main components in this particular alternator are two bistable vortex valves Fig. 18.

paths. The fluidic flow switching valves are also connected into the topologically square-shaped configuration of the two-phase "alternator" [21]. They switch the flows to alternate between the two inlet flowpaths $A-D$ and B-C. On top of the picturs are the two inlets of the clean and contaminated air, while at the bottom of these illustrations are the outlets leading through the exchangers into (and simultaneously and out from) the room. The frequency of flow switching may be adjusted by lengths of feedback channels, so that it is high enough for small size of the exchangers but low enough to avoid excessive contact between the two air flows.

An example of the actual circuit diagram realisation of the configurations from Figs. 20 and 21 is shown in the case of two switched vortex valves $A$ and $B$, discussed in [16] in the next Figs. 22 and 23. The switched valves $A$ and $B$ may be similar to the valve shown in Fig. 19. At the outlets from the vortex chambers are in the vertices $\mathrm{C}$ and $\mathrm{D}$ of the alternator "square" two passive valves - three-terminal fluidic elements with nozzle input (black triangles) and two outlet diffusers (white triangles). The diffusers are required to direct the incoming flow into the proper outlet. As in Figs. 20 and 21, the two flowpaths exchange their exit ends so that opening of the valve $A$ is contemporary with closed $B$ and vice versa. Connected to the axial diffuser is the heat storage matrix in the exhausts into the room. The next Fig. 23 indicates by the gray curved arrows the character of the air flows in one of the phases.

Finally, the last Fig. 24 shows than the apparent complexity of the circuit diagram presented in Fig. 22 does not have to correspond to tha actual complexity of the terminal unit. The bistable turn down valve in each flowpaths may be integrated with the 3-terminal valve ( which together form the alternator) into a compact unit.

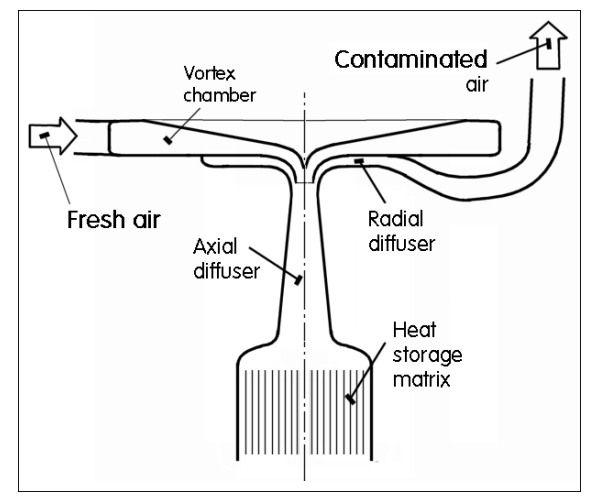

Fig. 24. Detail of the alternator from Fig. 21 with integral vortex valve and the three-terminal flow-directing element.

\section{Conclusions}

Heating his dwelling has been one of the oldest tasks that man was challenged to solve. In the earliest stages the solutions were almost trivial seen from present engineering point of view. Complications came with buildings containing several rooms - as it was quite early established that it is better to generate the heat in a central plant. This has brought the problem of optimising inside the building the heat transport. Real difficulties arose with the increasing emphasis on mutually contradictory requirements of economy and comfort. Paper discusses the solutions offered by terminal units operating without any mechanical moving components — using the fluid flow control ideas introduced recently by pure fluidics. Admittedly, designing fluidic devices themselves may be rather difficult (it is much easier to stop the air flow by a blocking mechanical component rather than by an aerodynamic effect). Nevertheless, fluidic terminal units have a huge potential for further developments. Their relative simplicity, reliability, and low cost make pure fluidic units suitable for use in large numbers per building, bringing the heat or cold up to the person's desk or workspace at optimum conditions -- at the same time saving by close local control a considerable proportion from the overall supplied thermal energy.

Author's stay during development of fluidic terminal units was supported by research grant Nr. 17-08218S obtained from the Czech Republic Grant Agency and also by institutional support RVO: 61388998. Author was led to several important ideas in discussions with Prof. K. Hemzal. 


\section{References}

1. V. Tesař.: Pressure-Driven Microfluidics, Monograph, Artech House Publishers, Norwood, MA 02062, USA, 2007

2. V. Tesař, H. Bandalusena, Experiments in Fluids, 50, 1225, 2011

3. R.W. Haines, D.C, Hittle, Control systems for heating, ventilating, and air conditioning", 6th edition, Springer 2006

4. H. Coanda: ,Device for deflecting a stream of elastic fluid projected into an elastic fluid“", Patent US 2052869

5. H.E. Steinacher, Die Kälte, 3, 107, 1969

6. V. Tesař : "Method of automatic control of air conditioning and means for performing it", Patent CZ175267

7. W. S. Ostrander: „Dual conduit air-conditioning system", US Patent 3623543,

8. V. Tesař: "Air inlet into an air-conditioned room", Patent CZ 185183

9. V. Tesař, EPJ Web of Conferences 92, 02096, 2015

10. V. Tesař, K. Hemzal, "Induction unit for air conditioning systems", Patent CZ 185179

11. G. Osheroff, "Induction terminal unit for airconditioning systems", Patent US 3799246

12. V. Tesař V.: "Noise of flowing fluid", Proc of Conf. Engineering Diagnostics VIII, ČSVTS FELČVUT, p. 37, Prague 1985
13. G. Clifford G.: „Modern heating, ventilating and air conditioning", Englewood Cliffs, Prentice Hall, N. J., 1984

14. R. W. Warren: „Fluid oscillator“. Patent US $3,016,066$.

15. V. Tesař, K. Hemzal K.: „Fluidic bistable power elements for terminal units of variable-volume air conditionings systems" Proc. of the Vth 'Jablonna' Fluidics Conference, Budapest, Hungary, 1974

16. V. Tesař : „, Heat-exhanger type ventilation”, Patent CZ180914.

17. V. Tesař: "Air inlet into a room for ventilation system with regeneration heat exchangers", Patent CZ180370

18. G. Walker, "Industrial heat exchangers: a basic guide" , Taylor \& Francis, Abingdon, United Kingdom, 1990

19. V. Tesař: „Ventilation system with regenerative use of waste heat ", Patent CZ 180798

20. V. Tesař V. EPJ Web of Conferences, 143, 02128, 2017

21. V. Tesař , Z. Trávníček, J. Kordík, Z. Randa, Sensors and Actuators A, 138, p. 213, 2007 